Published in final edited form as:

Gastroenterology. 2018 July ; 155(1): 107-117. doi:10.1053/j.gastro.2018.03.038.

\title{
Improved Diet Quality Associates With Reduction in Liver Fat- Particularly in Individuals With High Genetic Risk Scores for Nonalcoholic Fatty Liver Disease
}

\author{
Jiantao Ma1 ${ }^{1}$, Rachel Hennein ${ }^{1}$, Chunyu Liu ${ }^{1}$, Michelle T. Long ${ }^{2}$, Udo Hoffmann ${ }^{3}$, Paul F. \\ Jacques $^{4}$, Alice H. Lichtenstein ${ }^{5}$, Frank B. $\mathrm{Hu}^{6}$, and Daniel Levy ${ }^{1}$ \\ ${ }^{1}$ Population Sciences Branch, National Heart, Lung, and Blood Institute, National Institutes of \\ Health, Bethesda, Maryland and the Framingham Heart Study, Framingham, Massachusetts, \\ USA, 01702
}

${ }^{2}$ Section of Gastroenterology, Evans Department of Medicine, Boston University School of Medicine, Boston, Massachusetts, USA, 02118

${ }^{3}$ Radiology Department, Massachusetts General Hospital, Harvard Medical School, Boston, Massachusetts, USA, 02114

${ }^{4}$ Nutrition Epidemiology Program, USDA Human Nutrition Research Center on Aging at Tufts University, Boston, Massachusetts, USA, 02111

${ }^{5}$ Cardiovascular Nutrition Laboratory, USDA Human Nutrition Research Center on Aging at Tufts University, Boston, Massachusetts, USA, 02111

${ }^{6}$ Department of Nutrition, Harvard T.H. Chan School of Public Health, Boston, Massachusetts, USA, 02115

\footnotetext{
Correspondence to: Daniel Levy, MD, Framingham Heart Study, Framingham, MA, and the Population Sciences Branch, National Heart, Lung, and Blood Institute, National Institutes of Health, Bethesda, MD. levyd@nhlbi.nih.gov.

Publisher's Disclaimer: This is a PDF file of an unedited manuscript that has been accepted for publication. As a service to our customers we are providing this early version of the manuscript. The manuscript will undergo copyediting, typesetting, and review of the resulting proof before it is published in its final citable form. Please note that during the production process errors may be discovered which could affect the content, and all legal disclaimers that apply to the journal pertain.

Competing interest statement: All authors have completed the Unified Competing Interest form (available on request from the corresponding author) and declare: no support from any organization for the submitted work; no financial relationships with any organizations that might have an interest in the submitted work in the previous three years, no other relationships or activities that could appear to have influenced the submitted work.

Disclaimer: The views expressed in this manuscript are those of the authors and do not necessarily represent the views of the National Heart, Lung, and Blood Institute; the National Institutes of Health; or the U.S. Department of Health and Human Services.

Author contributions: JM and DL designed the analysis; JM analyzed the data; CL provided statistical support; and JM and RH wrote the manuscript; MTL, UH, PFJ, AHL, FBH, and DL provided critical editorial comments; DL had primary responsibility for the final content. All authors read and approved the final manuscript.

Availability of data and material: The datasets analyzed in the present study are available in the database of Genotypes and Phenotypes (https://www.ncbi.nlm.nih.gov/projects/gap/cgi-bin/study.cgi?study_id=phs0 00363.v15.p10).

Copyright: The corresponding author is a government employee and has the right to grant on behalf of all authors and does grant on behalf of all authors a non-exclusive license on a worldwide basis to the BMJ Publishing Group Ltd to permit this article (if accepted) to be published in BMJ editions and any other BMJPGL products and sublicenses such use and exploit all subsidiary rights, as set out in our license.

Transparency declaration: Corresponding Author affirms that the manuscript is an honest, accurate, and transparent account of the study being reported; that no important aspects of the study have been omitted; and that any discrepancies from the study as planned (and, if relevant, registered) have been explained.
} 


\section{Abstract}

Background \& Aims-Dietary modification has been recommended for treatment of nonalcoholic fatty liver disease (NAFLD), although it is not clear whether improving diet quality can prevent its development. We performed a prospective study to examine the association between diet quality change and change in liver fat change. We also examined the association between genetic risk score and liver fat change in individuals with different levels of diet quality change.

Methods-Our study included 1521 participants who attended the seventh and eighth examinations (1998-2001 and 2005-2008) of the second-generation cohort or attended the first and second examinations (2002-2005 and 2008-2011) of the third-generation cohort in the Framingham Heart Study. The self-administered semi-quantitative 126-item Harvard food frequency questionnaire was used to determine dietary intake in the year leading up to an examination. We assessed levels of liver fat using liver-phantom ratio (LPR) and computed tomography images from 2002 through 2005 and again from 2008 through 2011. LPR values are inversely related to liver fat-increased LPR indicates decreased liver fat. We examined associations of changes in 2 diet scores-the Mediterranean-style diet score (MDS) and Alternative Healthy Eating Index (AHEI) — with changes in liver fat and new-onset fatty liver. We evaluated interactions between diet score change and a weighted genetic risk score for NAFLD, determined based on multiple single nucleotide polymorphisms identified in genome-wide association studies of NAFLD. The primary outcome was change in LPR between baseline and follow-up measurement.

Results-For each 1-standard deviation increase in MDS, the LPR increased (meaning liver fat decreased) by 0.57 (95\% CI, $0.27-0.86 ; P<.001)$ and the odds for incident fatty liver decreased by $26 \%$ (95\% CI, 10\%-39\%; $P=.002$ ). For each 1-standard deviation increase in AHEI, LPR increased by 0.56 (95\% CI, $0.29-0.84 ; P<.001)$ and the odds for incident fatty liver decreased by $21 \%$ (95\% CI, 5\%-35\%; $P=.02$ ). Increased diet scores were also associated with reduced odds of developing more-advanced fatty liver. Higher genetic risk scores were associated with increased liver fat accumulation in participants who had decreased MDS $(P<.001)$ or AHEI scores $(P=.001)$, but not in those with stable or improved diet scores ( $P$ for gene-diet interaction <.001).

Conclusions-In an analysis of participants in the Framingham Heart Study, increasing diet quality, determined based on MDS and AHEI scores, is associated with less liver fat accumulation and reduced risk for new-onset fatty liver. An improved diet is particularly important for individuals with a high genetic risk for NAFLD.

\section{Keywords}

diet quality; fatty liver; steatosis; prevention

\section{Introduction}

In parallel with rising rates of obesity and type 2 diabetes, the occurrence of non-alcoholic fatty liver disease (NAFLD) has dramatically increased in recent decades to a worldwide ${ }^{1,2}$. The rise in NAFLD prevalence has important consequences; NAFLD is projected to become the primary indication for liver transplantation in the United States by $2025^{3,4}$. Given its 
correlation with cardiometabolic diseases, NAFLD has been posited as a target-organ manifestation of the metabolic syndrome ${ }^{5-9}$. Thus, prevention of NAFLD is important for public health.

Lifestyle modification, primarily a weight loss diet, is recommended for treatment of NAFLD $^{10}$. Available evidence also suggests that a healthy eating pattern such as a Mediterranean diet may favorably affect NAFLD independent of weight loss ${ }^{11,12}$. Prior population-based studies have utilized dietary scores developed to assess diet quality, such as the Mediterranean-style diet score (MDS) and the Alternative Healthy Eating Index (AHEI) score ${ }^{13,14}$. In general, adherence to favorable dietary patterns, captured by these scores, is associated with less weight gain, lower risk of incident cardiovascular disease, and decreased risk of death ${ }^{14-16}$. Adherence to a healthy diet is associated cross-sectionally with less hepatic fat ${ }^{17-19}$. The longitudinal association between diet quality and change in liver fat accumulation is unknown.

NAFLD is likely a consequence of the interaction between genetic predisposition and environmental, behavioral, and health factors including diet, diabetes, and obesity ${ }^{20}$. Genome-wide association studies (GWAS) have identified several NAFLD-associated loci ${ }^{21,22}$. A recent study also showed that body mass index (BMI), a measure of general adiposity, modifies the genetic effect on liver fat accumulation and that inter-individual variation in liver fat is partly determined by gene-environment interactions ${ }^{23}$. Nevertheless, studies examining the effect of gene-environment interactions on NAFLD are scarce. Therefore, the objectives of our study were to examine prospectively whether improved diet quality, assessed using two indices, MDS and AHEI score, is associated with a corresponding decline in liver fat accumulation, and to explore if diet quality modifies genetic risk for NAFLD.

\section{Methods}

\section{Study sample}

The study sample included participants from the second- and the third-generation cohorts of the Framingham Heart Study 24, 25. Briefly, 2,869 participants attended both the seventh and eighth examinations (1998-2001 and 2005-2008) of the Second Generation cohort and 3,411 participants attended both the first and second examinations (2002-2005 and 2008-2011) of the Third Generation cohort. A subgroup of these participants $(n=3,477)$ had liver fat measured at year of 2002 to 2005. Among these participants, 1,886 participants had also liver fat measured at year of 2008 to 2011 . We excluded participants if they had high alcohol consumption $(\mathrm{n}=144)$, defined as more than 21 drinks per week for men and more than 14 drinks per week for women ${ }^{10}$, or lacked dietary data $(n=96)$ or other covariates $(\mathrm{n}=125)$. Thus, a total of 1,521 participants (610 from the second-generation cohort and 911 from the third-generation cohort) were included for the primary analysis. The Framingham Heart Study protocols and procedures were approved by the Institutional Review Board for Human Research at Boston University Medical Center and all participants provided written informed consent. 


\section{Diet quality scores}

We used a previously validated, self-administered semi-quantitative 126-item Harvard food frequency questionnaire (FFQ) to determine dietary intake for the previous year leading up to an examination ${ }^{26}$. The FFQ was mailed to participants to be completed at home and returned during the study appointment. We excluded FFQ data when the reported energy intake was $<2.5 \mathrm{MJ} / \mathrm{d}(600 \mathrm{kcal} / \mathrm{d})$ for both men and women, $\geq 16.7 \mathrm{MJ} / \mathrm{d}(4000 \mathrm{kcal} / \mathrm{d})$ for women, $\geq 17.5 \mathrm{MJ} / \mathrm{d}(4200 \mathrm{kcal} / \mathrm{d})$ for men, and if $\geq 13$ food items were left blank. We calculated the MDS and AHEI scores at both baseline (Second Generation cohort exam seven; Third Generation cohort exam one) and follow-up (Second Generation exam eight; Third Generation cohort exam two).

The MDS had nine components (Supplemental Table 1): vegetables, fruits, nuts, legumes, whole grains, fish, red meat, ratio of monounsaturated fatty acids (MUFA) to saturated fatty acids (SFA), and alcohol ${ }^{13}$. Consumption of each food component was categorized into sex-, cohort-, and examination-specific quartiles. For all components except red meat and alcohol, the lowest to highest quartile categories were assigned scores of $0,1,2$, and 3 . The order of the scores was reversed for red meat. For alcohol, we assigned a score of 1 if consumption was $\geq 10$ grams/day and $\leq 25$ grams/day for men or $\geq 5$ grams/day and $\leq 15$ grams/day for women. We assigned an alcohol consumption score of 0 for all other values. All the scores were summed to create the MDS, which ranged from 0 to 25 , with higher scores indicating a healthier dietary pattern.

The AHEI was comprised of 11 components (Supplemental Table 1) including vegetables, fruits, nuts and legumes, sugar-sweetened beverages and fruit juice, whole grains, red meat, eicosapentaenoic acid (EPA) and docosahexaenoic acid (DHA), polyunsaturated fatty acids (PUFA without EPA and DHA), trans-fatty acids, sodium, and alcohol ${ }^{14}$. Scores for each component ranged from 0 to 10 , with a score of 10 indicating that the participant has met dietary recommendations based on the 2010 Dietary Guidelines for Americans. All scores were summed to create the AHEI score, comprehensively ranging from possible values of 0 to 110 .

\section{Liver fat}

The protocols for hepatic fat measurements in the Framingham Heart Study have been described elsewhere 27,28 . We used abdominal multidetector computed tomography (CT, General Electric Health Care) to measure liver fat. We conducted CT scans using an 8-slice scanner at baseline (2002-2005) and a 64-slice scanner at follow-up (2008-2011). To quantify liver fat, we calculated the mean Hounsfield units of three regions in the liver ${ }^{29}$. Then, the liver-phantom ratio (LPR) was calculated by dividing the mean Hounsfield units by the Hounsfield units of a 'phantom' control and multiplying by 100. LPR values are inversely related to liver fat content. We defined fatty liver as LPR less than or equal to the sex-, cohort-, and examination-cycle specific $30^{\text {th }}$ percentile of LPR (Supplemental Table 2) in participants without heavy alcohol consumption, previous myocardial infarction, stroke, cancer (except for non-melanoma skin cancer), or bariatric surgery. It has been shown that a serum aspartate aminotransferase (AST) to alanine aminotransferase (ALT) ratio $>1$ is associated with increased risk of advanced fibrosis in patients fatty liver ${ }^{30}$. Therefore, we 
further categorized our participants into the following mutually exclusive groups: no fatty liver, fatty liver alone (isolated NAFL), and fatty liver with serum AST/ALT ratio > 1 (advanced NAFL).

\section{Genetic risk score}

To create a genetic risk score (GRS), we first selected single nucleotide polymorphisms (SNPs) from published GWAS of NAFLD ${ }^{22,31,32}$. Selection criteria included: 1) GWAS in adults of European ancestry, 2) had replication or functional studies to validate genome-wide significant SNPs, and 3) minor allele frequency >0.05. For SNPs in LD (R-squared $\searrow 0.3$ ), we selected the variant with the lowest P-value in GWAS. Five SNPs (rs738409, rs2228603, rs12137855, rs780094, and rs4240624) from one published GWAS meta-analysis of NAFLD were selected ${ }^{22}$. Functional features of these SNPs and mapped genes are summarized in Supplemental Table 3. Genotyping in the Framingham Heart Study was performed with the Affymetrix 550K Array ${ }^{33}$. SNPs were imputed to the 1000 Genomes Project reference panel $^{34}$. Genotypes were coded using continuous dosages from 0 to 2 . We then developed a weighted GRS by summing together the product of the number of NAFLD-associated risk alleles and the corresponding regression coefficient (Supplemental Table 3) derived from the GWAS $^{22}$.

\section{Covariate assessments}

We assessed all covariates when participants visited the research clinic in accordance with standard protocols ${ }^{35}$. Body mass index (BMI) was calculated as weight divided by height squared $\left(\mathrm{kg} / \mathrm{m}^{2}\right)$. Waist circumference was measured at the level of the umbilicus with the participant in a standing position. Current smokers were defined as participants who selfreported smoking at least one cigarette per day in the prior year. We used self-reported consumption of beer, wine, liquor, and spirits to determine alcohol drinking ${ }^{36}$. We generated a physical activity score using the intensity and time spent for each type of activity assessed by the physical activity questionnaire 37 .

\section{Statistical analysis}

The primary exposures were change in MDS ( $\triangle \mathrm{MDS}$ ) and change in AHEI score ( $\triangle \mathrm{AHEI})$ and the primary outcome was LPR change ( $\triangle \mathrm{LPR}$ ) between baseline and follow-up. We applied linear mixed models to account for the family structure in our study sample. The initial model (Model 1) adjusted for sex, age, and baseline dietary score (i.e., baseline MDS when exposure was $\triangle \mathrm{MDS}$ and baseline AHEI score when exposure was $\triangle \mathrm{AHEI}$ ), baseline LPR, baseline AST/ALT ratio, energy intake, smoking status, and physical activity score. In Model 2, we additionally adjusted for baseline BMI and changes in energy intake, smoking status, and physical activity score. We next adjusted for BMI change in Model 3. We also conducted a mediation test to estimate the proportion of mediation by $\Delta \mathrm{BMI}$. In the mediation test, we used multiple linear regression models and nonparametric bootstrap with 1000 simulations to estimate confidence intervals. To explore which specific dietary components might drive the association, we applied a similar statistical approach for changes in the individual components of MDS and AHEI between baseline and follow-up. In these analyses, instead of adjusting for baseline dietary score, we adjusted for baseline score of each individual component. 
We examined the prospective association of diet change ( $\triangle \mathrm{MDS}$ and $\triangle \mathrm{AHEI}$ ) and incident fatty liver at follow-up. We used generalized estimating equations to estimate the odds ratio (OR) of incident fatty liver. In addition, we calculated ORs of incident fatty liver as a function of diet change with and without the inclusion of elevated AST/ALT ratio using logistic regression models with the generalized logit link function. The cumulative logit link function in logistic regression models was used to evaluate the linear trend associated with diet change. In the analyses of incident fatty liver, we excluded participants with fatty liver at baseline. We adjusted for the same covariates as described above in the primary analyses.

To study gene-diet interactions, we stratified participants into tertile categories according to their GRS, i.e., participants who carried low, medium, and high genetic risk for developing NAFLD. We also stratified participants into tertile categories according to $\triangle \mathrm{MDS}$ and $\triangle$ AHEI. We then performed stratified analyses to examine the associations between GRS and $\triangle \mathrm{LPR}$ in each stratum of the dietary score change using linear mixed models with adjustment for the same covariates as described in the primary analysis. We formally examined the interaction by testing the multiplicative term of the GRS and $\triangle \mathrm{MDS}$ or GRS and $\triangle \mathrm{AHEI}^{38,39}$.

We conducted a sensitivity analysis by repeating the primary analyses after additionally excluding participants with a history of bariatric surgery, myocardial infarction, stroke, and cancer (except for non-melanoma skin cancer) before the follow-up examination. Waist circumference is an independent risk factor for fatty liver. In the present sample, waist circumference was highly correlated with BMI (Pearson $r=0.9$ ). We then repeated the primary analyses by adjusting for baseline and change in waist circumference instead of baseline BMI and $\triangle \mathrm{BMI}$. We also conducted analyses to account for potential differences between the two studied cohorts. For example, CT scans were obtained near the time of the research clinic examinations for the Third Generation cohort, whereas CT scans were conducted about 3 years after the research clinic examinations for the Second Generation cohort. Thus, we repeated the abovementioned analyses in each cohort separately. Another major difference between the two cohorts was age. We therefore performed an interaction analysis by testing the significance of the product of $\triangle$ MDS or $\triangle$ AHEI with age group dichotomized at the median value ( $<49$ years or $\geq 49$ years). Interaction between sex and change in dietary scores was also examined.

All statistical analyses were conducted using SAS statistical software (version 9.3; SAS Institute, Cary, North Carolina) or R statistical analysis software (version 3.3.3; https:// www.R-project.org). Given the exploratory nature of the present study, we considered a twotailed $\mathrm{p}<0.05$ value as statistically significant.

\section{Results}

\section{Participant characteristics}

As shown in Table 1, from baseline to follow-up (median interval six years), mean BMI increased by $0.7 \mathrm{~kg} / \mathrm{m}^{2}$, mean waist circumference increased by $3.1 \mathrm{~cm}$, and the mean LPR decreased by 1.1. The dietary scores calculated at baseline and at follow-up examinations were correlated, Pearson $r$ was 0.60 for MDS and 0.65 for AHEI (both $\mathrm{P}<0.001$ ). However, a 
large inter-individual variation was also observed, mean (standard deviation; SD) was 0.3 (4.0) for $\triangle \mathrm{MDS}$ and 6.0 (10.3) for $\triangle \mathrm{AHEI}$ (Table 1, Supplemental Tables 4 and 5).

\section{Change in diet quality and change in BMI and waist circumference}

After adjusting for multiple covariates (Supplemental Table 6 and 7), both serially increased (i.e. healthier) MDS and AHEI score were associated with less weight gain. For one SD increase in MDS, BMI declined by $0.38 \mathrm{~kg} / \mathrm{m}^{2}(95 \% \mathrm{CI}: 0.24,0.51 ; \mathrm{P}<0.001)$ and WC declined by $1.07 \mathrm{~cm}(95 \% \mathrm{CI}: 0.69,1.45 ; \mathrm{P}<0.001)$. Similarly, per SD increase in AHEI score, BMI declined by $0.38 \mathrm{~kg} / \mathrm{m}^{2}$ (95\%CI: $\left.0.25,0.51 ; \mathrm{P}<0.001\right)$ and $\mathrm{WC}$ declined by 0.99 cm (95\%CI: 0.64, 1.35; $\mathrm{P}<0.001)$.

\section{Change in diet quality and change in LPR}

As shown in Table 2, increased MDS score from baseline to follow-up was associated with reduced liver fat accumulation after adjusting for baseline values and longitudinal changes in covariates; for each SD serial increase in MDS, the decline in LPR during followup (an indicator of increased liver fat accumulation) decreased by 0.57 (95\% CI: 0.27, 0.86; $\mathrm{P}<0.001$ ). LPR declined the most, -1.90 (95\%CI: $-2.63,-1.18$ ), in the lowest quartile category of $\triangle \mathrm{MDS}$ (median MDS decreased by 4 units). In contrast, change in LPR (-0.49; $95 \%$ CI: $-1.19,0.21$ ) was not significantly different from zero in the highest quartile category of $\triangle \mathrm{MDS}$ (median MDS increased by 4 units). After also adjusting for $\triangle \mathrm{BMI}$, the association between $\triangle \mathrm{MDS}$ and $\triangle \mathrm{LPR}$ was attenuated, but remained statistically significant ( $\mathrm{P}=0.02$ ). The proportion of mediation by $\Delta \mathrm{BMI}$ was $38 \%$ (95\% CI: $19 \%-73 \% ; \mathrm{P}<0.001$ ).

We observed similar associations between $\triangle \mathrm{AHEI}$ and $\triangle \mathrm{LPR}$ (Table 3). For each SD serial increase in AHEI score, the corresponding decline in LPR was 0.56 (95\%CI: 0.29, 0.84; $\mathrm{P}<0.001$ ) after adjusting for baseline values and changes in covariates. Participants in the lowest quartile category of $\triangle$ AHEI (median AHEI decrease by 6 units) had the greatest reductions in LPR $(-1.71$; $95 \% \mathrm{CI}:-2.41,-1.01)$, while, those in the highest quartile category of $\triangle \mathrm{AHEI}$ (median AHEI increased by 18 units) had least reduction in LPR ( -0.34 ; $95 \% \mathrm{CI}:-1.07,0.38)$. The association between $\triangle \mathrm{AHEI}$ and $\triangle \mathrm{LPR}$ also remained significant after additional adjustment for $\triangle \mathrm{BMI}(\mathrm{P}=0.01)$. The proportion of mediation by $\triangle \mathrm{BMI}$ was $38 \%$ (95\%CI: $22 \%-73 \%$; $\mathrm{P}<0.001)$.

\section{Change in diet quality and incident fatty liver}

We observed that both increased MDS and increased AHEI were associated with reduced risk of incident fatty liver after adjusting for baseline values and changes in covariates (Figure 1A and 1B). The odds of incident fatty liver declined by 26\% (95\%CI: 10\%, 39\%; $\mathrm{P}=0.002)$ for one SD increase in MDS and by $21 \%$ (95\%CI: $5 \%, 35 \%$; $\mathrm{P}=0.02)$ for one SD increase in AHEI. Odds ratios (ORs) for incident fatty liver for each quartile category of $\triangle \mathrm{MDS}$ and $\triangle \mathrm{AHEI}$ are shown in Supplemental Table 8 . After additionally adjusting for $\triangle \mathrm{BMI}$ (Supplemental Table 7), the observed association remained significant for $\triangle \mathrm{MDS}$ $(\mathrm{P}=0.03)$ but not for $\triangle \mathrm{AHEI}(\mathrm{P}=0.12)$.

Figure 2 shows that both the risks of incident isolated NAFL and advanced NAFL declined in concert with an increase in MDS or AHEI. For one SD increase in MDS, OR of incident 
isolated NAFL was 0.77 (95\% CI: 0.60, 0.97; $\mathrm{P}=0.03$ ) and $\mathrm{OR}$ of incident advanced NAFL was 0.68 (95\% CI: 0.49, 0.94; $\mathrm{P}=0.02$ ), $\mathrm{P}$-trend $=0.002$. For one SD increase in AHEI, OR for incident isolated NAFL was 0.80 (95\%CI: $0.64,0.99 ; \mathrm{P}=0.049)$ and $\mathrm{OR}$ for incident advanced NAFL was 0.76 (95\%CI: 0.56, 1.03; P=0.07), P-trend=0.01.

\section{Change in individual dietary components and change in liver fat}

Among the nine components of MDS, increased scores for vegetables, nuts, legumes, and red meat (a higher score indicating a higher intake of vegetables, nuts, and legumes and a lower intake for red meat) were associated with less liver fat accumulation after adjusting for baseline values and longitudinal changes in covariates (Supplemental Table 9). Each 1-SD increase in the scores for vegetables, nuts, legumes, and red meat were associated with 0.31 (95\%CI: 0.001, 0.62, P=0.049), 0.41 (95\%CI: 0.09, 0.74, $\mathrm{P}=0.01$ ), 0.38 (95\%CI: 0.07, 0.69, $\mathrm{P}=0.02)$, and 0.35 (95\%CI: $0.02,0.67, \mathrm{P}=0.04)$ increases in $\mathrm{LPR}$, respectively, reflecting a longitudinal decline in liver fat.

Among the 11 components of the AHEI, increased scores for vegetables, fruits, nuts and legumes, whole grains, red meat, EPA and DHA, and trans-fatty acids (a higher score indicating a higher intake of vegetables, fruits, nuts and legumes, whole grains, and EPA and DHA and a lower intake for red meat and trans-fatty acids) were associated with lower liver fat accumulation after adjusting for baseline values and changes in covariates (Supplemental Table 10). Each 1-SD increase for these seven individual scores was associated with 0.36 (95\%CI: 0.06, 0.67, P=0.02), 0.50 (95\% CI: 0.20, 0.80, P=0.001), 0.39 (95\%CI: 0.09, 0.69, $\mathrm{P}=0.01), 0.34$ (95\%CI: 0.02, 0.66, $\mathrm{P}=0.04), 0.37$ (95\%CI: 0.04, 0.69, $\mathrm{P}=0.03), 0.31$ (95\%CI: $0.02,0.60, \mathrm{P}=0.03$ ), 0.41 (95\%CI: $0.08,0.75, \mathrm{P}=0.02$ ) increases in LPR, respectively.

In addition, the combined fruit and vegetable scores in both MDS and AHEI were inversely associated with liver fat accumulation, $\mathrm{P}=0.02$ and $\mathrm{P}=0.01$ (Supplemental Table 9 and 10). After additionally adjusting for $\triangle \mathrm{BMI}$, the nuts score in MDS and the score for nuts and legumes in AHEI remained significant $(\mathrm{P}=0.02$ and $\mathrm{P}=0.04$, respectively). For both MDS and AHEI, the strength of the association between individual components and $\triangle \mathrm{LPR}$ was generally lower than that for the overall score.

\section{Gene-diet interaction for change in LPR}

The interaction term between GRS and $\triangle$ MDS was significant ( $\mathrm{P}<0.001$; Figure $3 \mathrm{~A})$. Among participants who were in the lowest tertile category of $\triangle \mathrm{MDS}$ (i.e. declining diet quality from baseline to follow-up) we observed a significant association between NAFLDassociated GRS and increased liver fat accumulation from baseline to follow-up ( $\mathrm{P}<0.001)$. In contrast, the association between GRS and $\triangle \mathrm{LPR}$ was not significant in participants who were in the middle and highest tertile categories of $\triangle \mathrm{MDS}$, i.e. those who had a stable or improved diet quality. The interaction term was also significant between GRS and $\triangle \mathrm{AHEI}$ $(\mathrm{P}<0.001$; Figure 3B). We observed a similar relationship for $\triangle \mathrm{AHEI}$; an inverse association between GRS and $\triangle \mathrm{LPR}$ was only significant in participants in the lowest tertile category of $\triangle \mathrm{AHEI}$, representing those with declining diet quality from baseline to follow-up ( $\mathrm{P}=0.001)$. In participants whose diet remained stable or improved, the association between GRS and $\triangle$ LPR was not significant. 


\section{Sensitivity analysis}

After excluding 168 participants with a history of bariatric surgery, myocardial infarction, stroke, or cancer (except for non-melanoma skin cancer) before the follow-up examinations, the association remained similar between $\triangle \mathrm{MDS}$ and $\triangle \mathrm{LPR}$ (Supplemental Table 11) and between $\triangle$ AHEI and $\triangle$ LPR (Supplemental Table 12). Substitution of waist circumference for BMI did not substantially alter the observed associations between $\triangle \mathrm{MDS}$ and $\triangle \mathrm{LPR}$ (Supplemental Table 13) or between $\triangle$ AHEI and $\triangle$ LPR (Supplemental Table 14). As shown in Supplemental Figure 1, the observed overall association between change in diet quality ( $\triangle \mathrm{MDS}$ and $\triangle \mathrm{AHEI}$ ) and $\triangle \mathrm{LPR}$ was driven by the significant association in the Third Generation cohort, a younger group in which dietary assessment, clinical examinations, and liver fat measurements were assessed at the same time. Further, we observed no interaction between age and change in dietary scores $(\mathrm{P}=0.40$ for $\triangle \mathrm{MDS}$ and $\mathrm{P}=0.09$ for $\triangle \mathrm{AHEI})$ or between sex and change in dietary scores $(\mathrm{P}=0.82$ for $\triangle \mathrm{MDS}$ and $\mathrm{P}=0.62$ for $\triangle \mathrm{AHEI})$.

\section{Discussion}

From our longitudinal analysis we observed that improved diet quality from baseline to follow-up was associated with reduced liver fat accumulation in a group of middle-aged to older adults. Compared with participants whose diet quality declined most over time (i.e. the lowest quartile of longitudinal dietary score change), those whose diet improved the most (i.e. the highest quartile) had about $80 \%$ less liver fat accumulation from baseline to followup. We found that these associations were largely driven by increased consumption of fruits, vegetables, nuts, legumes, whole grains, EPA and DHA and decreased consumption of red meat and trans-fat. We also observed that adopting a healthier eating pattern was associated with lower risk of developing fatty liver, particularly fatty liver accompanied by biochemical changes (increased AST/ALT ratio). Furthermore, we found that adopting a healthier diet might mitigate an individual's genetic predisposition to NAFLD. Considered in aggregate, these findings indicate that after accounting for serial weight change, the adoption of a healthier diet is associated with favorable changes in liver fat accumulation and reduced risk of NAFLD.

The present study examined two a priori dietary scores, MDS and AHEI ${ }^{13}{ }^{14}$. Whereas most of the individual MDS dietary components overlapped with the AHEI components, the scores assigned to each component were weighted differently; MDS used population-based cutoffs, whereas AHEI was constructed based on dietary guidelines. Therefore, the two scores complement each other.

A recent prospective observational study of 146,071 individuals, who were followed for up to 20 years, demonstrated that increases in MDS and AHEI were associated with less weight gain ${ }^{40}$. Our findings on the relations of dietary scores to longitudinal change in weight were similar; we found that after six years of observation, improved diet quality scores were associated with a lower increase in BMI and waist.

To our knowledge, ours is the first prospective study to examine the relations of long-term habitual diet to fatty liver. A cross-sectional study among 5,079 participants enrolled in a multi-ethnic cohort showed that a higher diet-quality score, constructed based on the 
Mediterranean diet, was associated with less liver fat ${ }^{41}$. Further, a six-week randomized, crossover intervention study implemented in 12 biopsy-proven NAFLD cases demonstrated that, compared with a low-fat and high-carbohydrate diet, a Mediterranean diet was associated with greater reductions in liver fat despite similar weight loss between the two diets $^{11}$.

The present study extends the current literature by providing novel evidence of an inverse association between long-term diet quality changes and serial changes in liver fat accumulation in the general population.

Previous studies have shown that dietary constituents, such as omega-6/omega-3 PUFA ratio and dietary carbohydrate, might modify the effect of a NAFLD-associated polymorphism, rs738409 (PNPLA3), on liver fat accumulation in adolescents ${ }^{42,43}$. However, studies examining the ways in which overall diet quality may interact with genetic risk factors for NAFLD are lacking. Our findings indicate that worsening diet quality may accentuate the genetic predisposition to NAFLD, while improving diet quality may mitigate genetic risk. Replication studies in independent cohorts with sufficient sample sizes are needed to validate our observations.

Our observations suggest that two types of mechanisms may be involved in the relationship between diet and liver fat. First, diet may impact liver fat deposition by regulating overall adiposity. This is supported by our findings that the association of diet quality change and liver fat accumulation was attenuated by adjusting for change in BMI. Several food components such as fruits and vegetables may affect liver fat through this mechanism. Fruits and vegetables contain high amounts of water and fiber, which can decrease energy intake by affecting satiety and improve weight control ${ }^{44}$. High fiber consumption may promote enrichment of certain bacteria in the gut such as members in phylum Firmicutes ${ }^{45}$, which is commonly depleted in NAFLD patients ${ }^{46}$. One potential pathway linking dietary fiber, gut microbiota, and NAFLD is through regulating the bacterial synthesis of short-chain fatty acids (SCFAs) ${ }^{46}$. SCFAs are hypothesized to play complex roles in NAFLD. High fiber consumption increases the richness of fiber-degrading bacteria, which in turn increase the production of beneficial SCFAs such as butyrate. Available evidence reveals that butyrate may suppress inflammation and thus reduce the likelihood of the development and progression of NAFLD ${ }^{46}$. The other mechanism that could link diet to liver fat accumulation is based on specific biochemical processes occurring in the liver. This hypothesis is supported by our observation that increased nut or combined nut and legume consumption was associated with reduced liver fat accumulation independent of change in BMI. Preliminary evidence suggests that nut consumption may promote fat oxidation and reduced ectopic fat mass in viscera ${ }^{47}$. However, as human studies supporting this hypothesis are limited, future research exploring the benefits of nut consumption on hepatic fat accumulation is warranted.

NALFD has reached epidemic-levels and has a substantial impact on public health ${ }^{1}$. NAFLD is associated with increased type 2 diabetes risk ${ }^{9,48}$. It is also projected to become the leading indication for liver transplantation in the US by $2025^{3,4}$, and when simple hepatic steatosis progresses to nonalcoholic steatohepatitis (NASH), individuals are at an increased 
risk for liver cancer ${ }^{49}$. Our findings emphasize the notion that improving diet quality is an important strategy for NAFLD prevention and, particularly among those with high genetic risk.

The methods used in this study have many strengths, particularly the prospective assessment of changes in diet and liver fat with six years of observations. There are also several limitations that warrant discussion. We used an 8-slice scanner at baseline (2002-2005) and a 64-slice scanner at follow-up (2008-2011). The technology differed from the baseline to the follow-up examination, which may have impacted absolute values of the LPR. For this reason, we used percentiles rather than fixed values of LPR to define changes in liver fat accumulation and fatty liver. In the second-generation cohort, CT was conducted on average three years after the clinical examinations, which might bias point estimates toward a weaker observed association. The AST to ALT ratio has been examined as a surrogate marker for advanced liver fibrosis, but sensitivity of the ratio is relatively low ${ }^{30}$. Because we lacked additional data, we did not explore liver fat changes related to viral infection and use of medications such as steroids and tamoxifen. However, we believe that fatty liver due to these exposures is rare in our study setting. We selected several key dietary components to construct the MDS and AHEI, however, other dietary constituents may play important roles in liver fat metabolism. Longitudinal change in dietary scores observed in the present study might reflect a regression to mean phenomenon; however, this phenomenon would likely attenuate the observed associations. The semi-quantitative FFQ used in the present study may lead to misclassification. In addition, diet was not assessed between baseline and follow-up examinations. Whereas we adjusted for a variety of lifestyle factors, residual confounders cannot be ruled out. Finally, our participants are predominantly white, which limits the generalizability of these results to other populations.

We demonstrate that improved diet quality over six years was associated with reduced liver fat accumulation and lower risk and severity of fatty liver in a group of middle-aged to older adults. Our findings also indicate that improved diet quality may be particularly important for those with high genetic risk for NAFLD. Future intervention studies are needed to test the efficacy and efficiency of diet-based approaches for NAFLD prevention as well as to examine mechanisms underlying the association between diet and NAFLD.

\section{Supplementary Material}

Refer to Web version on PubMed Central for supplementary material.

\section{Acknowledgments}

Funding: This work was supported by the National Heart, Lung and Blood Institute's Framingham Heart Study (Contract No. N01-HC-25195) and its contract with Affymetrix for genotyping services (Contract No. N02HL-6-4278). Several authors are employees of the National Heart, Lung and Blood Institute and their research is supported by its Division of Intramural Research (JM, RH, CL, DL).

\section{References}

Author names in bold designate shared co-first authorship 
1. Rinella ME. Nonalcoholic fatty liver disease: a systematic review. JAMA. 2015; 313:2263-73. [PubMed: 26057287]

2. Williams CD, Stengel J, Asike MI, et al. Prevalence of nonalcoholic fatty liver disease and nonalcoholic steatohepatitis among a largely middle-aged population utilizing ultrasound and liver biopsy: a prospective study. Gastroenterology. 2011; 140:124-31. [PubMed: 20858492]

3. Wong RAM, Cheung R, Perumpail RB, Harrison SA, Younossi ZM, Ahmed A. Nonalcoholic steatohepatitis is the second leading etiology of liver disease among adults awaiting liver transplantation in the United States. Gastroenterology. 2015; 148:547-55. [PubMed: 25461851]

4. Charlton M. Nonalcoholic fatty liver disease: a review of current understanding and future impact. Clin Gastroenterol Hepatol. 2004; 2:1048-58. [PubMed: 15625647]

5. Targher G, Byrne CD, Lonardo A, et al. Non-alcoholic fatty liver disease and risk of incident cardiovascular disease: A meta-analysis. J Hepatol. 2016; 65:589-600. [PubMed: 27212244]

6. Hamaguchi M, Kojima T, Takeda N, et al. Nonalcoholic fatty liver disease is a novel predictor of cardiovascular disease. World J Gastroenterol. 2007; 13:1579-84. [PubMed: 17461452]

7. Feitosa MF, Reiner AP, Wojczynski MK, et al. Sex-influenced association of nonalcoholic fatty liver disease with coronary heart disease. Atherosclerosis. 2013; 227:420-4. [PubMed: 23390892]

8. Speliotes EK, Massaro JM, Hoffmann U, et al. Fatty liver is associated with dyslipidemia and dysglycemia independent of visceral fat: the Framingham Heart Study. Hepatology. 2010; 51:1979_ 87. [PubMed: 20336705]

9. Ma J, Hwang SJ, Pedley A, et al. Bi-directional analysis between fatty liver and cardiovascular disease risk factors. J Hepatol. 2017; 66:390-97. [PubMed: 27729222]

10. Chalasani N, Younossi Z, Lavine JE, et al. The Diagnosis and Management of Nonalcoholic Fatty Liver Disease: Practice Guidance from the American Association for the Study of Liver Diseases. Hepatology. 2018; 67:328-57. [PubMed: 28714183]

11. Ryan MC, Itsiopoulos C, Thodis T, et al. The Mediterranean diet improves hepatic steatosis and insulin sensitivity in individuals with non-alcoholic fatty liver disease. J Hepatol. 2013; 59:138-43. [PubMed: 23485520]

12. Zelber-Sagi S, Ratziu V, Oren R. Nutrition and physical activity in NAFLD: an overview of the epidemiological evidence. World J Gastroenterol. 2011; 17:3377-89. [PubMed: 21876630]

13. Fung TT, Rexrode KM, Mantzoros CS, et al. Mediterranean diet and incidence of and mortality from coronary heart disease and stroke in women. Circulation. 2009; 119:1093-100. [PubMed: 19221219]

14. Chiuve SE, Fung TT, Rimm EB, et al. Alternative dietary indices both strongly predict risk of chronic disease. J Nutr. 2012; 142:1009-18. [PubMed: 22513989]

15. Fung TT, Pan A, Hou T, et al. Long-Term Change in Diet Quality Is Associated with Body Weight Change in Men and Women. J Nutr. 2015; 145:1850-6. [PubMed: 26084363]

16. Sotos-Prieto M, Bhupathiraju SN, Mattei J, et al. Association of Changes in Diet Quality with Total and Cause-Specific Mortality. N Engl J Med. 2017; 377:143-53. [PubMed: 28700845]

17. Koch M, Nothlings U, Lieb W. Dietary patterns and fatty liver disease. Curr Opin Lipidol. 2015; 26:35-41. [PubMed: 25501880]

18. Velasco N, Contreras A, Grassi B. The Mediterranean diet, hepatic steatosis and nonalcoholic fatty liver disease. Curr Opin Clin Nutr Metab Care. 2014; 17:453-7. [PubMed: 25023188]

19. Zelber-sagi S, Salomone F, Mlynarsky L. The Mediterranean dietary pattern as the diet of choice for non-alcoholic fatty liver disease: Evidence and plausible mechanisms. Liver Int. 2017; 37:93649. [PubMed: 28371239]

20. Anstee QM, Seth D, Day CP. Genetic Factors That Affect Risk of Alcoholic and Nonalcoholic Fatty Liver Disease. Gastroenterology. 2016; 150:1728-44. [PubMed: 26873399]

21. Eckel RH, Jakicic JM, Ard JD, et al. 2013 AHA/ACC guideline on lifestyle management to reduce cardiovascular risk: a report of the American College of Cardiology/American Heart Association Task Force on Practice Guidelines. Circulation. 2014; 129:S76-99. [PubMed: 24222015]

22. Speliotes EK, Y-A LM, Wu J, Hernaez R, Kim LJ, et al. Genome-wide association analysis identifies variants associated with nonalcoholic fatty liver disease that have distinct effects on metabolic traits. PLoS Genet. 2011; 7:e1001324. [PubMed: 21423719] 
23. Stender S, Kozlitina J, Nordestgaard BG, Tybjærg-hansen A, Hobbs HH, Cohen JC. Adiposity amplifies the genetic risk of fatty liver disease conferred by multiple loci. Nat Genet. 2017; 49:842-7. [PubMed: 28436986]

24. Splansky GL, Corey D, Yang Q, Atwood LD, Cupples LA, Benjamin EJ, D'Agostino RB Sr, Fox CS, Larson MG, Murabito JM, O'Donnell CJ, Vasan RS, Wolf PA, Levy D. The Third Generation Cohort of the National Heart, Lung, and Blood Institute's Framingham Heart Study: design, recruitment, and initial examination. Am J Epidemiol. 2007; 165:1328-35. [PubMed: 17372189]

25. Kannel WB, Feinleib M, McNamara PM, Garrison RJ, Castelli WP. An investigation of coronary heart disease in families. The Framingham offspring study. Am J Epidemiol. 1979; 110:281-90. [PubMed: 474565]

26. Rimm EB, Giovannucci EL, Stampfer MJ, Colditz GA, Litin LB, Willett WC. Reproducibility and validity of an expanded self-administered semiquantitative food frequency questionnaire among male health professionals. Am J Epidemiol. 1992; 135:1114-26. [PubMed: 1632423]

27. Speliotes EK, Massaro MJ, Hoffmann U, Foster MC, Sahani DV, Hirschhorn JN, O’Donnell CJ, Fox CS. Liver fat is reproducibly measured using computed tomography in the Framingham Heart Study. J Gastroenterol Hepatol. 2008; 23:894-9. [PubMed: 18565021]

28. Ma J, Hwang SJ, Pedley A, Massaro JM, Hoffmann U, Chung RT, Benjamin EJ, Levy D, Fox CS, Long MT. Bi-directional analysis between fatty liver and cardiovascular disease risk factors. J Hepatol. 2017; 66:390-7. [PubMed: 27729222]

29. Speliotes EK, Massaro MJ, Hoffmann U, et al. Liver fat is reproducibly measured using computed tomography in the Framingham Heart Study. J Gastroenterol Hepatol. 2008; 23:894-9. [PubMed: 18565021]

30. McPherson S, Stewart SF, Henderson E, et al. Simple non-invasive fibrosis scoring systems can reliably exclude advanced fibrosis in patients with non-alcoholic fatty liver disease. Gut. 2010; 59:1265-9. [PubMed: 20801772]

31. Kozlitina J, Smagris E, Stender S, et al. Exome-wide association study identifies a TM6SF2 variant that confers susceptibility to nonalcoholic fatty liver disease. Nat Genet. 2014; 46:352-6. [PubMed: 24531328]

32. Chalasani N, Guo X, Loomba R, et al. Genome-wide association study identifies variants associated with histologic features of nonalcoholic Fatty liver disease. Gastroenterology. 2010; 139:1567-76. [PubMed: 20708005]

33. Ma J, Yang Q, Hwang SJ, Fox CS, Chu AY. Genetic risk score and risk of stage 3 chronic kidney disease. BMC Nephrol. 2017; 18:32. [PubMed: 28103844]

34. Genomes Project C. Auton A, Brooks LD, et al. A global reference for human genetic variation. Nature. 2015; 526:68-74. [PubMed: 26432245]

35. Preis SR, Pencina MJ, Hwang SJ, et al. Trends in cardiovascular disease risk factors in individuals with and without diabetes mellitus in the Framingham Heart Study. Circulation. 2009; 120:21220. [PubMed: 19581493]

36. Walsh CR, Larson MG, Evans JC, et al. Alcohol consumption and risk for congestive heart failure in the Framingham Heart Study. Ann Intern Med. 2002; 136:181-91. [PubMed: 11827493]

37. Kannel WB, Belanger A, D'agostino R, Israel I. Physical activity and physical demand on the job and risk of cardiovascular disease and death: the Framingham Study. Am Heart J. 1986; 112:8205. [PubMed: 3766383]

38. Tyrrell J, Wood AR, Ames RM, et al. Gene-obesogenic environment interactions in the UK Biobank study. Int J Epidemiol. 2017; 46:559-75. [PubMed: 28073954]

39. Qi Q, Chu AY, Kang JH, et al. Sugar-sweetened beverages and genetic risk of obesity. N Engl J Med. 2012; 367:1387-96. [PubMed: 22998338]

40. Fung TT, Pan A, Hou T, et al. Long-Term Change in Diet Quality Is Associated with Body Weight Change in Men and Women. J Nutr. 2015; 145:1850-6. [PubMed: 26084363]

41. Shah RV, Murthy VL, Allison MA, et al. Diet and adipose tissue distributions: The Multi-Ethnic Study of Atherosclerosis. Nutr Metab Cardiovasc Dis. 2016; 26:185-93. [PubMed: 26899879]

42. Santoro N, Savoye M, Kim G, et al. Hepatic fat accumulation is modulated by the interaction between the rs 738409 variant in the PNPLA3 gene and the dietary omega6/omega3 PUFA intake. PLoS One. 2012; 7:e37827. [PubMed: 22629460] 
43. Davis JN, Le KA, Walker RW, et al. Increased hepatic fat in overweight Hispanic youth influenced by interaction between genetic variation in PNPLA3 and high dietary carbohydrate and sugar consumption. Am J Clin Nutr. 2010; 92:1522-7. [PubMed: 20962157]

44. Rolls BJ, Ello-Martin JA, Tohill BC. What can intervention studies tell us about the relationship between fruit and vegetable consumption and weight management? Nutr Rev. 2004; 62:1-17. [PubMed: 14995052]

45. De Filippis F, Pellegrini N, Vannini L, et al. High-level adherence to a Mediterranean diet beneficially impacts the gut microbiota and associated metabolome. Gut. 2016; 65:1812-21. [PubMed: 26416813]

46. Leung C, Rivera L, Furness JB, et al. The role of the gut microbiota in NAFLD. Nat Rev Gastroenterol Hepatol. 2016; 13:412-25. [PubMed: 27273168]

47. Tan SY, Dhillon J, Mattes RD. A review of the effects of nuts on appetite, food intake, metabolism, and body weight. Am J Clin Nutr. 2014; 100(Suppl 1):412S-22S. [PubMed: 24920033]

48. Anstee QM, Targher G, Day CP. Progression of NAFLD to diabetes mellitus, cardiovascular disease or cirrhosis. Nat Rev Gastroenterol Hepatol. 2013; 10:330-44. [PubMed: 23507799]

49. Michelotti GA, Machado MV, Diehl AM. NAFLD, NASH and liver cancer. Nat Rev Gastroenterol Hepatol. 2013; 10:656-65. [PubMed: 24080776] 
A

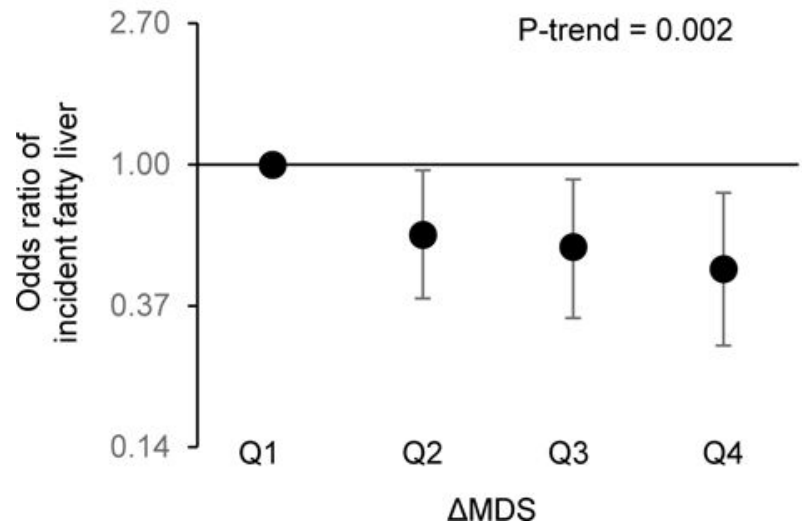

B

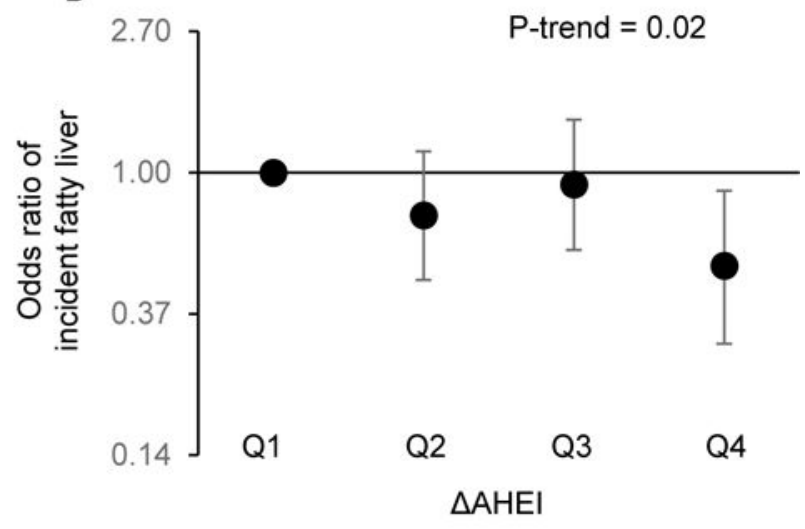

Figure 1.

Association of change in Mediterranean-style diet ( $\triangle \mathrm{MDS}$ ) and change in alternative healthy eating index ( $\triangle \mathrm{AHEI})$ and incident fatty liver. Symbols and bars are odds ratios and $95 \%$ confidence interval. 1A is for $\triangle \mathrm{MDS}$ and $1 \mathrm{~B}$ is for $\triangle \mathrm{AHEI}$. Model adjusted for sex, age, baseline MDS or AHEI, baseline liver-phantom ratio, baseline AST/ALT ratio, energy intake, smoking, physical activity level, BMI, as well as change in smoking status, physical activity, and energy intake. 


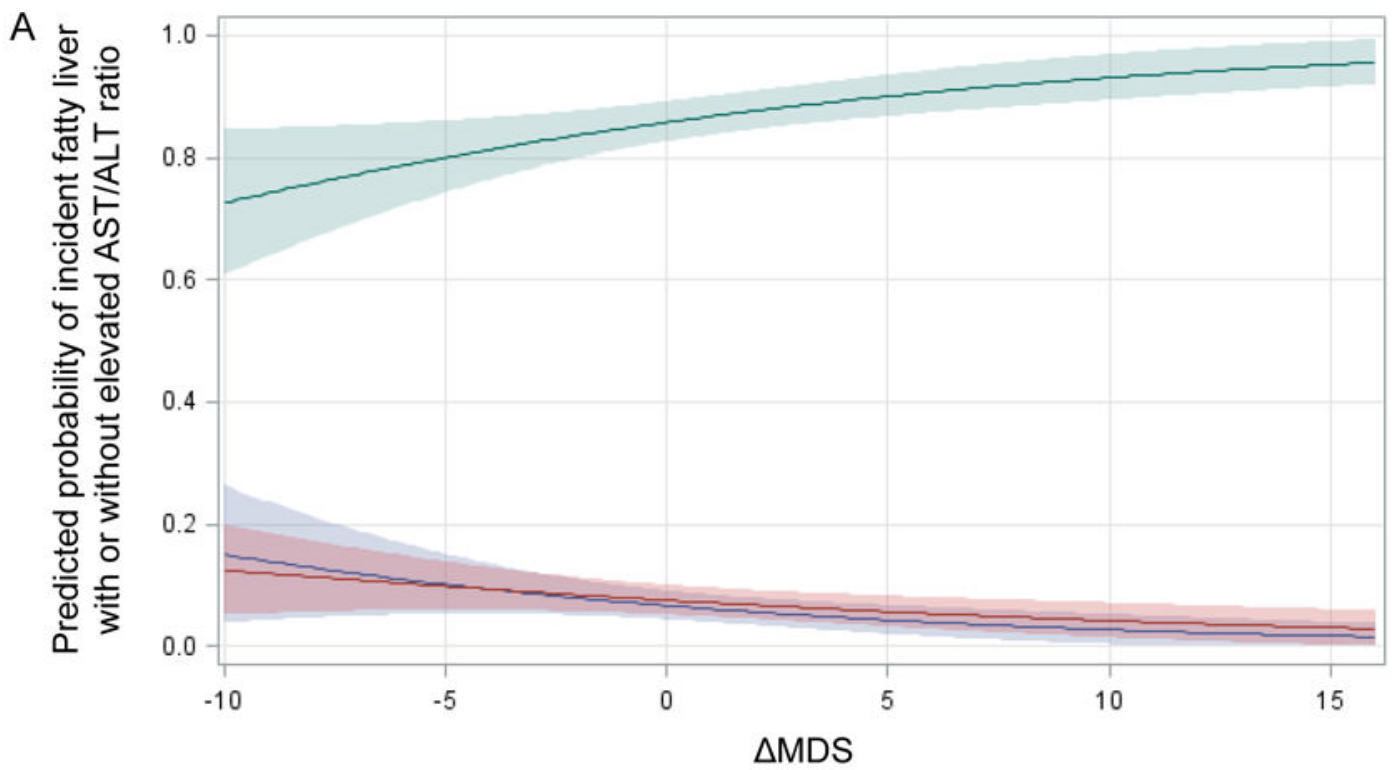

B

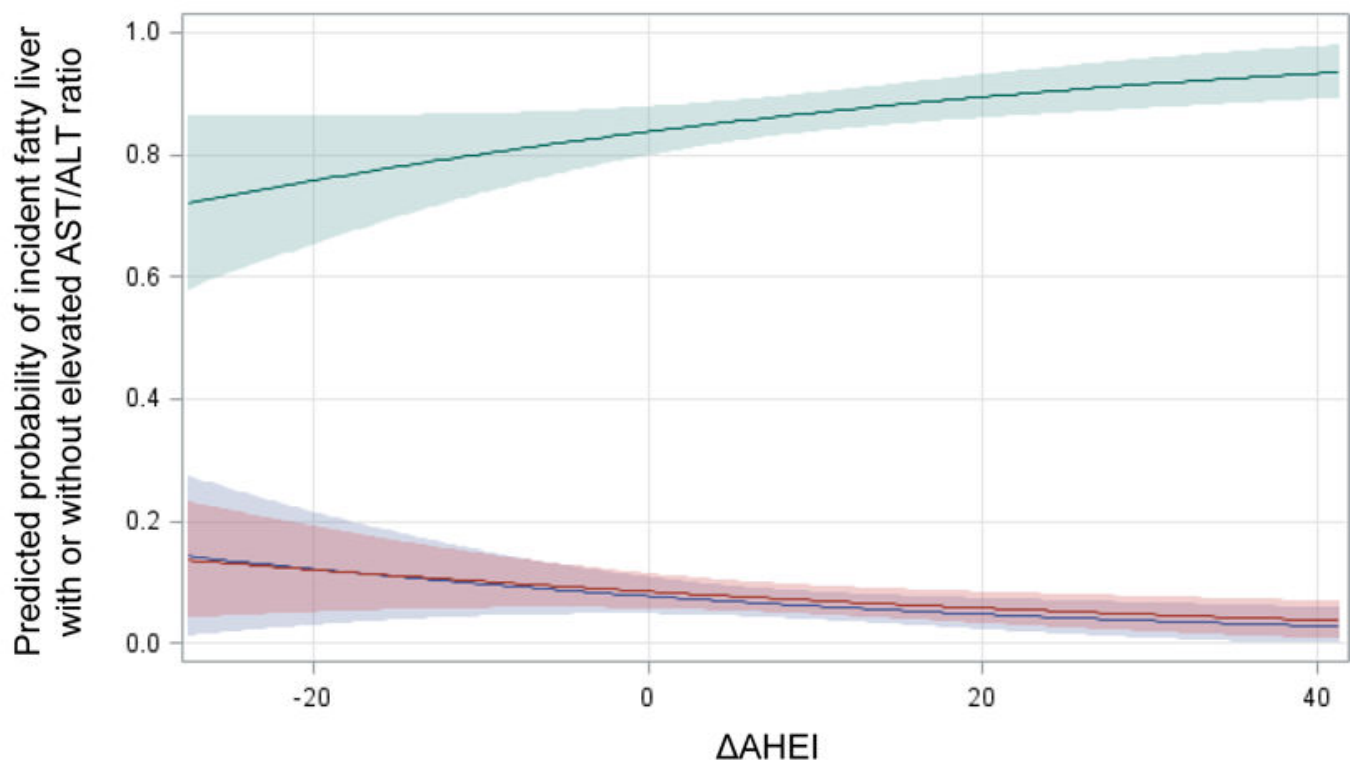

No fatty liver $\quad$ Isolated NAFL _ Advanced NAFL

Figure 2.

Predicted probability of incident fatty liver with elevated AST/ALT ratio (advanced NAFL) and incident fatty liver without elevated AST/ALT ratio (isolated NAFL) according to the change of diet quality. 2A for the Mediterranean-style dietary score (MDS) and 2B for the alternative healthy eating index (AHEI). Sample size was 1092, no fatty liver (N=912), isolated NAFL $(\mathrm{N}=122)$, and advanced NAFL $(\mathrm{N}=58)$. Model adjusted for sex, age, baseline MDS or AHEI, baseline liver-phantom ratio, baseline AST/ALT ratio, and baseline values of energy intake, smoking, physical activity level, BMI, as well as change in smoking status, physical activity, and energy intake. 


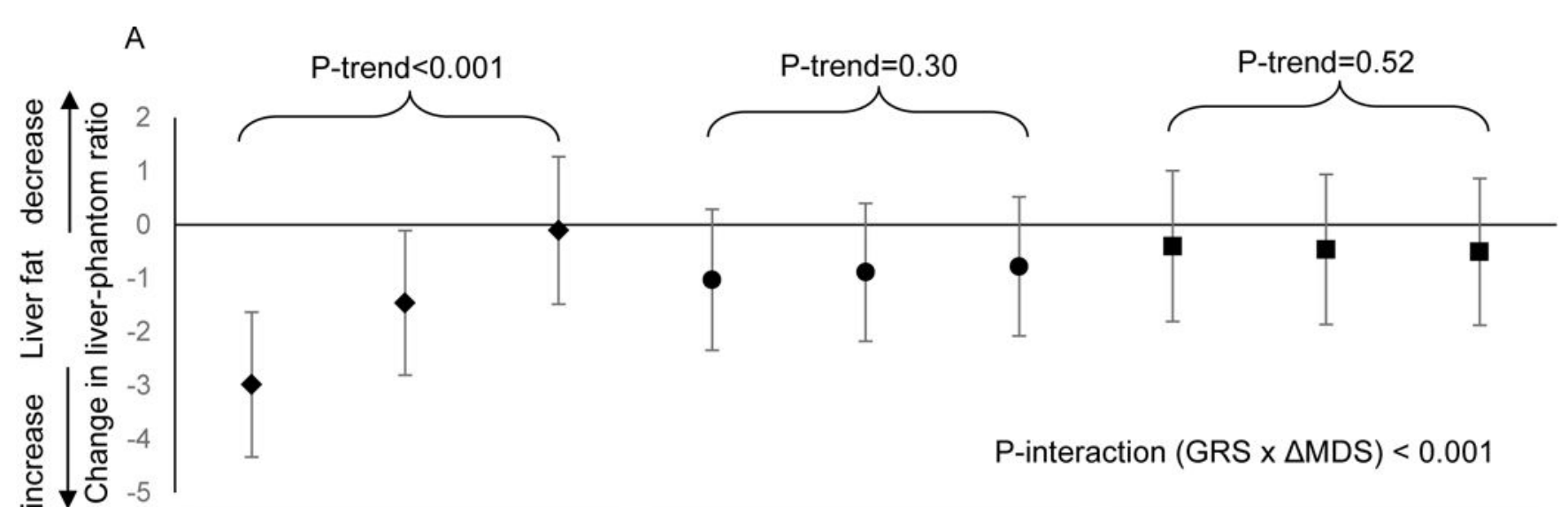

Genetic risk score
High Medium Low

MDS change $\mathrm{T} 1$

(Median MDS change -4)

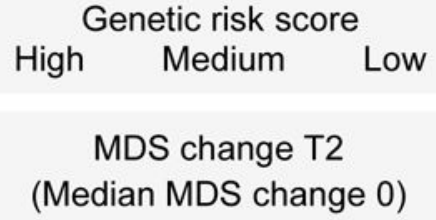

High Medium Low

(Median MDS change 0)
High Medium Low

MDS change T3

(Median MDS change 4)

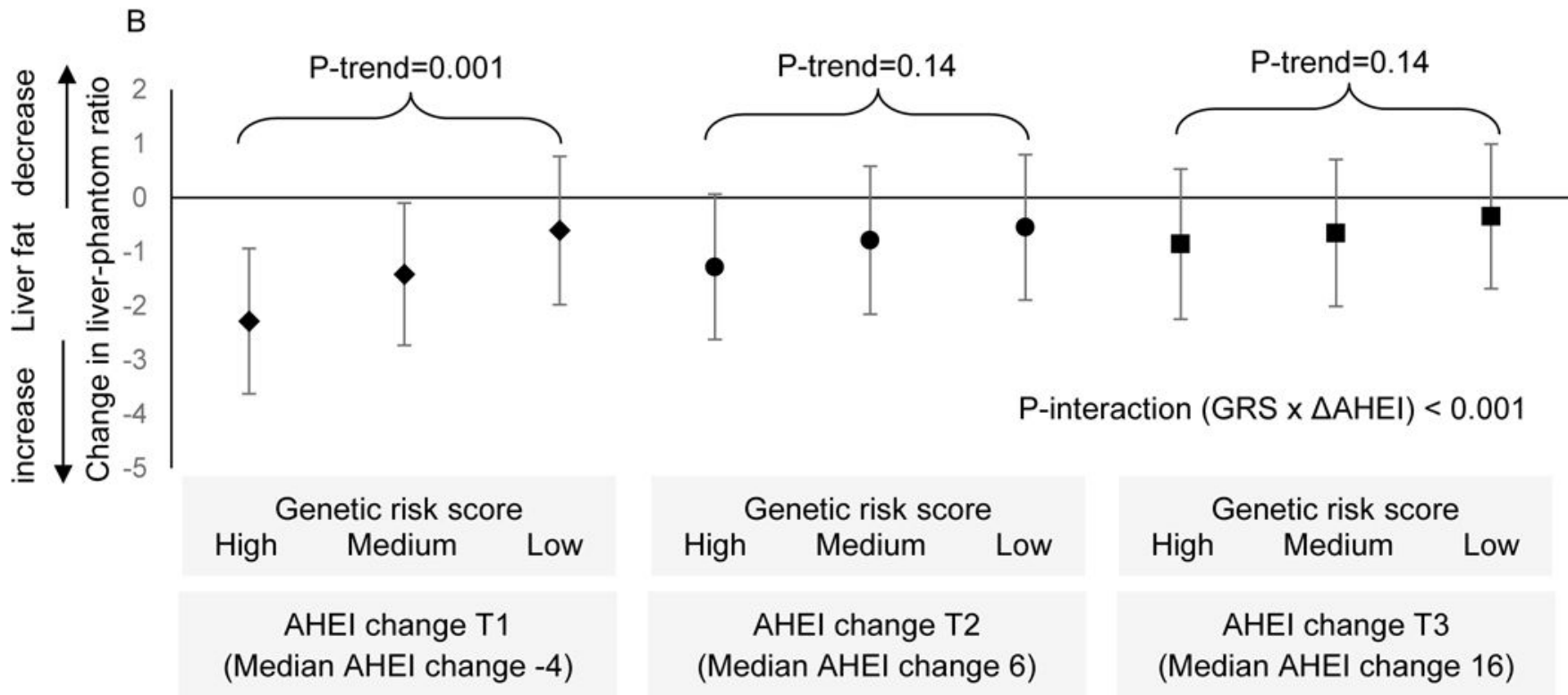

Figure 3.

Association between genetic risk score (GRS; tertile categories) and change in liverphantom ratio across tertile categories of dietary score change. A is for Mediterranean-style dietary score (MDS) and B is for alternative healthy eating index (AHEI). Values of y-axis are mean change and $95 \%$ confidence interval in liver-phantom ratio (a lower value represent increase in liver fat accumulation). P-interaction and P-trend were calculated with adjustment for sex, age, and baseline values of liver-phantom ratio, dietary score (MDS or AHEI), aspartate aminotransferase (AST)/alanine aminotransferase (ALT) ratio, smoking 
status, physical activity level, energy intake, BMI, as well as change in smoking status, physical activity, and energy intake. 